\title{
Inverse Linear Multistep Methods for the Numerical Solution of Initial Value Problems of Ordinary Differential Equations
}

\author{
By Peter Alfeld
}

\begin{abstract}
The well-known explicit linear multistep methods for the numerical solution of ordinary differential equations advance the numerical solution from $x_{n+k-1}$ to $x_{n+k}$ by computing some numerical approximation from back values and then evaluating the problem defining function to obtain an approximation of the derivative. In this paper similar methods are proposed that first compute an approximation to the derivative and then compute an approximation to the exact solution, either by evaluating a suitable function, or by solving a nonlinear system of equations. The methods can be applied to initial value problems where the exact solution is explicitly given in terms of the derivative. They can also be applied in the context of the CDS technique for certain stiff initial value problems of ordinary differential equations, introduced in [1] and [2]. Local accuracy and stability of the methods are defined and investigated, and specific methods, containing free parameters, are given.

The methods are not convergent, but they yield very good numerical results if applied to the type of problem they are designed for. Their major advantage is that they significantly reduce the amount of implicitness necessary in the numerical solution of certain problems.
\end{abstract}

1. Introduction. Consider the initial value problem (IVP)

$$
F\left(x, y, y^{\prime}\right)=0 ; \quad y(a)=\eta ; \quad y, F \in \mathbf{R}^{m} ; \quad x \in[a, b] .
$$

Special cases of (1.1) are

$$
y^{\prime}=f(x, y) ; \quad y(a)=\eta ; \quad y, f \in \mathbf{R}^{m} ; \quad x \in[a, b],
$$

and

$$
y=g\left(x, y^{\prime}\right) ; \quad y^{\prime}(a)=\omega ; \quad y, g \in \mathbf{R}^{m} ; \quad x \in[a, b] .
$$

An example for a physical problem, arising naturally in the theory of viscoelasticity, that gives rise to an initial value problem of the form (1.3) is described in [2] .

Sometimes (1.2) can be transformed into (1.3), e.g. in the case that

$$
f(x, y)=\lambda y ; \quad \lambda \in \mathbf{R} ; \quad \lambda \neq 0,
$$

where the corresponding problem (1.3) is given by

$$
g\left(x, y^{\prime}\right)=\lambda^{-1} y^{\prime} ; \quad \omega=\lambda^{-1} \eta .
$$

Received November 22, 1977

AMS (MOS) subject classifications (1970). Primary 65 L05. 
On the other hand, the simple integration problem defined by

$$
f(x, y)=p(x)
$$

with some function $p$ cannot be transformed into (1.3). Whenever it is necessary, we shall assume that (1.2) can be transformed into (1.3) and vice versa.

The problem (1.2) can be solved numerically using the explicit linear multistep method (ELMM)

$$
\sum_{j=0}^{k} \alpha_{j} y_{n+j}=h \sum_{j=0}^{k-1} \beta_{j} f_{n+j}
$$

where $\alpha_{n}=1, y_{n+j}$ is an approximation to $y\left(x_{n+j}\right)$ (with $x_{n}=a+n h, n=0,1,2$, $\ldots, h$ is a given constant, the step-length), and $f_{n}=f\left(x_{n}, y_{n}\right), n=0,1,2, \ldots$, (see [6, Chapter 2]). We ignore the problem of finding the starting values $y_{0}, y_{1}$, $\ldots, y_{k-1}$.

Similarly, (1.3) can be solved numerically by defining

$$
h^{-1} \sum_{j=0}^{k-1} \hat{\alpha}_{j} y_{n+j}=\sum_{j=0}^{k} \hat{\beta}_{j} f_{n+j}
$$

where $\hat{\beta}_{k}=1$ and $y_{n+j}=g\left(x_{n+j}, f_{n+j}\right)$.

The equation (1.5) defines a class of methods, whose properties are investigated in this paper. We refer to these methods as inverse linear multistep methods (ILMM).

It is assumed that the reader is familiar with the theory of linear multistep methods as it is developed in [6]. A knowledge of [1] is desirable, and essential for the understanding of Sections 6 and 7 of this paper.

2. Local Accuracy. With the ILMM (1.5) we associate the difference operator:

$$
T(z(x), h):=-\sum_{j=0}^{k}\left(h^{-1} \hat{\alpha}_{j} z\left(x_{n+j}\right)-\hat{\beta}_{j} z^{\prime}\left(x_{n+j}\right)\right),
$$

where $z(x)$ is an arbitrarily often differentiable test function. Proceeding similarly as for ELMMs, we expand $T(z(x), h)$, collect terms, and obtain

$$
T(z(x), h)=h^{-1} \hat{C}_{0} z(x)+\hat{C}_{1} z^{(\mathrm{i})}(x)+\hat{C}_{2} z^{(\mathrm{ii})}(x)+\cdots,
$$

where the $\hat{C}_{q}$ are given by

$$
\begin{aligned}
& \hat{C}_{0}=\hat{\alpha}_{0}+\hat{\alpha}_{1}+\cdots+\hat{\alpha}_{k}, \\
& \hat{C}_{q}=\frac{1}{q !} \sum_{j=0}^{k} j^{q} \hat{\alpha}_{j}-\frac{1}{(q-1) !} \sum_{j=0}^{k} j^{q-1} \hat{\beta}_{j}, \quad q=1,2,3, \ldots,
\end{aligned}
$$

where $0^{0}:=1$, and $\hat{\alpha}_{k}:=0$.

Remark. Here we proceed similarly as is done for ELMMs (see [6, p. 23]). The $\hat{C}_{i}$ are formally the same as the $C_{i}$ in [6], except that the coefficients of the ELMM are replaced by those of the ILMM.

Definition 1. The local truncation error of the inverse linear multistep method (1.5) at $x_{n+k}$ is defined to be the expression $T\left(y\left(x_{n}\right), h\right)$ given by (2.2), where $y(x)$ is the exact solution of the initial value problem (1.3). 
Remark. Thus, for ELMMs (respectively, ILMMs) the local truncation error is given by the difference $y\left(x_{n+k}\right)-y_{n+k}$ (respectively $\left.y^{\prime}\left(x_{n+k}\right)-f_{n+k}\right)$, provided the back values used for the computation of $y_{n+k}$ (respectively, $f_{n+k}$ ) are exact.

Definition 2. The inverse linear multistep method (1.5) is said to be of order $p$, if, in (2.2):

$$
\hat{C}_{0}=\hat{C}_{1}=\cdots=\hat{C}_{p+1}=0, \quad \hat{C}_{p+2} \neq 0
$$

$\hat{C}_{p+2}$ is called the error constant of (1.5), which is said to be consistent if it is of order $p \geqslant 1$.

Remarks. Note that in the corresponding definition (see [6]) for linear multistep methods we have that $C_{p+1} \neq 0$. This difference is due to the factor $h^{-1}$ in (1.5).

It is easily verified that $\hat{C}_{p+2}$ is independent of the point the local truncation error is expanded about (see [6, p. 24]). Thus, both ELMMs and ILMMs are of order $p$ if the local truncation error is of exact order $h^{p+1}$.

For a $k$-step method (1.5) we have $2 k$ parameters at our disposal and can set to zero $2 k$ of the coefficients given by (2.3). Thus, we can expect a maximum order $2 k$ - 2 for (1.5). Note that a one-step ILMM cannot be consistent.

Linear multistep methods of maximum order are of little use in practice because they are not zero-stable, and, as we shall see, something similar happens for inverse linear multistep methods.

3. Stability of Inverse Linear Multistep Methods. The stability of ELMMs is governed by their stability polynomial. The same applies to ILMMs.

Definition 3. The polynomials

$$
\begin{aligned}
& \zeta(r)=\sum_{j=0}^{k} \hat{\alpha}_{j} r^{j}, \quad \hat{\sigma}(r)=\sum_{j=0}^{k} \hat{\beta}_{j} r^{j}, \\
& \hat{\Pi}(r, \bar{h})=\hat{\zeta}(r)-\bar{h} \hat{\sigma}(r) ; \quad \bar{h} \in \mathbf{C},
\end{aligned}
$$

are said to be the first and second characteristic polynomials and the stability polynomial of (1.5), respectively.

Similarly, as for LMMs we define

Definition 4. The inverse linear multistep method (1.5) is said to be absolutely stable for a given $\bar{h} \in \mathrm{C}$ if, for that $\bar{h}$, all the roots $r_{t}$ of (3.1) satisfy $\left|r_{t}\right|<1, t=1$, $2, \ldots, k$, and to be absolutely unstable for that $\bar{h}$, otherwise. The set $R:=\{\bar{h} \in \mathbf{C} \mid$ (1.5) is absolutely stable for $\bar{h}\}$ is called the region of absolute stability of (1.5), the set $\mathrm{C}-\mathrm{R}$ is called the region of absolute instability of (1.5).

For simplicity we restrict ourselves to the case where the zeros of $\hat{\Pi}(x, \bar{h})$ and $\Pi(x, \bar{h})$ are distinct. If there are multiple roots, similar reasoning applies (cf. [5, p. 213]).

If either an ELMM or an ILMM is applied to the test equation

$$
y^{\prime}=\lambda y ; \quad y, \lambda \in \mathbf{C}
$$


we obtain the general numerical solutions

$$
y_{n}=\sum_{j=1}^{k} \hat{\gamma}_{j} \hat{\zeta}_{j}^{n}
$$

and

$$
y_{n}=\sum_{j=1}^{k} \gamma_{j} \xi_{j}^{n},
$$

respectively, where the $\hat{\gamma}_{j}$ and $\gamma_{j}$ are arbitrary constants, and the $\hat{\zeta}_{j}$ and $\zeta_{j}$ are the roots of the stability polynomials of the ILMM and the ELMM, respectively.

It follows that (as for the case of ELMMs) the ILMM (1.5) is absolutely stable for a given $h \lambda \in \mathbf{C}$, if all numerical solutions $\left\{y_{n}\right\}$ of (3.2) by (1.5) with step-length $h$ tend to zero as $n$ tends to infinity.

Here we encounter the fundamental peculiarity of ILMMS: for $h \lambda=0, \hat{\Pi}(r, h \lambda)$ is of degree $k-1$, and thus possesses only $k-1$ roots. This corresponds to the fact that if $h \lambda=0(1.5)$ cannot be applied to (3.2) at all, because (3.2) cannot be transformed into (1.3) (if $\lambda=0$ ), or (1.5) itself does not make sense (if $h=0$, i.e. $h^{-1}$ $=\infty)$.

The major application area of ILMMs are situations in which $h \lambda$ is large and negative, i.e. where we want all numerical solutions of (1.3) by (1.5) to tend to zero as $n$ tends to infinity. This is equivalent to requiring that the zeros of $\hat{\Pi}(r, h \lambda)$ are less than one in modulus for sufficiently large $h$.

Since the zeros of the stability polynomial $\hat{\Pi}(r, \bar{h})$ tend to those of $\hat{\sigma}(r)$ as $|\bar{h}|$ tends to infinity (cf. [5, p. 236]), we are led to seek ILMMs whose second characteristic polynomial possesses only zeros of modulus $<1$, i.e. is a Schur polynomial.

Definition 5. The inverse linear multistep method (1.5) is said to be infinitestable, if $\hat{\sigma}(r)$ is a Schur polynomial.

Remark. The concept of infinite-stability is in a way dual to the concept of zero-stability (cf. [6, p. 33] , [5, p. 218]). Zero-stability deals with the case that $h \lambda$ tends to zero. Infinite-stability deals with the case that $|h \lambda|$ tends to infinity. The practical interpretation of this is not so straightforward, because $h$ cannot tend to infinity for a practical problem on a finite interval, and $\lambda$ is independent on the IVP. However, we can consider a family of IVPs

$$
y^{\prime}=\lambda(y-z(x))+z^{\prime}(x) ; \quad \eta=z(a)
$$

where the exact solution $z(x)$ is independent of the parameter $\lambda$, which tends to infinity (in modulus).

The exact solution of the test equation (3.2) tends to zero faster, the larger $-\lambda$ (for $\lambda \in \mathbf{R}$ ). We would like this property to be reproduced by the ILMM. If some zeros of $\hat{\Pi}(r, \bar{h})$ are bounded away from zero (as $|\bar{h}|$ tends to infinity), the numerical solution will in general not tend faster to zero than $\gamma^{n}$, where $\gamma \neq 0$ is some constant independent of $h$. If, however, all zeros of $\hat{\Pi}(r, \bar{h})$ tend to zero (as $|\bar{h}|$ tends to infinity), the rate with which the numerical solution tends to zero can be arbitrarily high. 
Since the roots of $\hat{\Pi}(r, \bar{h})$ tend to those of $\sigma(r)$ (as $|\bar{h}|$ tends to infinity), we define

Definition 6. The inverse linear multistep method (1.5) is said to be strongly infinite-stable if $\sigma(r)=r^{k}$.

Remarks. For a strongly infinite-stable ILMM (1.5) becomes

$$
f_{n+k}=h^{-1} \sum_{j=0}^{k-1} \hat{\alpha}_{j} y_{n+j} .
$$

For a strongly infinite-stable ILMM we have $k$ parameters $\hat{\alpha}_{0}, \hat{\alpha}_{1}, \ldots, \hat{\alpha}_{k-1}$ at our disposal and can, thus, expect to be able to attain order $k-2$. A consistent strongly infinite-stable ILMM has a step-number $k \geqslant 3$.

Strong infinite-stability resembles $L$-stability (see [3]). If a strongly infinitestable ILMM is applied to (1.5) with starting values $y_{0}, y_{1}, \ldots, y_{k-1}$, and steplength $h$, independent of $\lambda$, then

$$
\lim _{\lambda \rightarrow-\infty} y_{k}=0
$$

Obviously strong infinite-stability implies infinite-stability.

The following theorem gives the maximum attainable orders of infinite-stable and strongly infinite-stable ILMMs.

THEOREM. (a) Let $\hat{\sigma}(r)$ be a polynomial of degree $k\left(\right.$ with $\left.\hat{\beta}_{k}=1\right)$. Then there exists a unique polynomial $\hat{\zeta}(r)$ of degree $k-1$, such that the inverse linear multistep method defined by $\hat{\sigma}(r)$ and $\hat{\zeta}(r)$ has order at least $k-2$.

(b) The maximum order of an infinite-stable inverse linear multistep method is $k-2$. For each $k \geqslant 3$ there exists a strongly infinite-stable inverse linear multistep method of order $k-2$.

Proof. (Note: the proof follows closely the lines of Henrici's [5] proof on the maximum order of zero-stable LMMs. The roles played by the first and second characteristic polynomial are interchanged, and some adjustments have to be made to account for the term $h^{-1}$ in the expansion (2.2) of the local truncation error, and for the fact that by definition the second characteristic polynomial does not possess zeros of modulus 1 , whereas zero-stability allows for such zeros.)

With an ILMM we associate the function

$$
\psi(\xi):=\hat{\zeta}(\xi)-\ln (\xi) \sigma(\xi)
$$

Then (1.15) is of order $p$ if $\psi(\xi)$ possesses a zero of exact order $p+2$ at $\xi=1$.

To see this, assume that the exact solution of the invertible IVP, to which (1.5) is to be applied, is given by $y(x)=e^{x}$. Suppose (1.5) is of order $p$. Substituting into (2.2) and assuming $x_{n}=0$ yields

$$
h^{-1} \hat{\zeta}\left(e^{h}\right)-\hat{\sigma}\left(e^{h}\right)=O\left(h^{p+1}\right)
$$

or

$$
\hat{\zeta}\left(e^{h}\right)-h \hat{\sigma}\left(e^{h}\right)=O\left(h^{p+2}\right)
$$


Setting $\xi=e^{h}$ this becomes

$$
\hat{\zeta}(\xi)-\ln (\xi) \hat{\sigma}(\xi)=\psi(\xi)=O\left(\xi^{p+2}\right),
$$

i.e. $\psi(\xi)$ has a zero of order $p+2$ at $\xi=1$. Conversely, assume $\psi(\xi)$ has a zero of order $p+2$, i.e. (3.5) holds. This implies (3.4) and because the order of (1.5) depends on the coefficients $\hat{\alpha}_{j}$ and $\hat{\beta}_{j}$ only, the ILMM is of order $p$ (cf. [5, pp. 225, 226]).

To prove the first part of the theorem observe that the function $\ln (\xi) \hat{\sigma}(\xi)$ is holomorphic at $\xi=1$ and can, thus, be expanded about $\xi=1$, i.e. there exist coefficients $g_{j}, j=0,1,2, \ldots$, such that

$$
\ln (\xi) \hat{\sigma}(\xi)=g_{0}+g_{1}(\xi-1)+g_{2}(\xi-1)^{2}+\cdots .
$$

Define

$$
\hat{\zeta}(\xi)=g_{0}+g_{1}(\xi-1)+\cdots+g_{k-1}(\xi-1)^{k-1} .
$$

With this definition $\psi(\xi)$ has a zero of order $k$ at $\xi=1$ and, thus, (1.5) is of order $k-2$ (cf. [5, p. 226]).

We now turn to part (b) of the theorem. Assume $\sigma(r)$ is a Schur polynomial.

To handle this assumption we use the transformation

$$
z=(\xi-1) /(\xi+1), \quad \xi=(1+z) /(1-z)
$$

(cf. [5, p. 229]), which maps the unit circle into the left half plane, and define

$$
\begin{aligned}
& r(z):=(1-z)^{k} \hat{\zeta}\left((1+z)^{\prime} /(1-z)\right), \\
& s(z):=(1-z)^{k} \sigma((1+z) /(1-z)) .
\end{aligned}
$$

We have

$$
s(z)=a_{0}+a_{1} z+\cdots+a_{k} z^{k}
$$

where the $a_{j}, j=0,1, \ldots, k$, are real. Because $\sigma(\zeta)$ is a Schur polynomial, the imaginary parts of $s(z)$ are all strictly negative. This implies that all coefficients of $s(z)$ are nonzero and of the same sign (cf. [5, p. 230]). Define further

$$
\begin{aligned}
p(z) & :=(1-z)^{k} \psi((1+z) /(1-z)) \\
& =r(z)-\ln ((1+z) /(1-z)) s(z) .
\end{aligned}
$$

The function $p(z)$ has a zero of order $p+2$ at $z=0(\xi=1)$ if $(1.5)$ is of order $p$. Consequently, if $(1.5)$ is of order $p$, then

$$
r(z)=b_{0}+b_{1} z+\cdots+b_{p+1} z^{p+1},
$$

where

$$
\ln ((1+z) /(1+z)) s(z)=b_{0}+b_{1} z+b_{2} z^{2}+\cdots .
$$

(Note that $\ln ((1+z) /(1-z)) s(z)$ is holomorphic at $z=0$.) 
Since the degree of $r(z)$ must not exceed $k-1$, the existence of an ILMM of order $p>k-2$ implies that

$$
b_{k}=b_{k+1}=\cdots=b_{p+1}=0 .
$$

We show that for an infinite-stable ILMM $b_{k} \neq 0$. We obtain

$$
\ln ((1+z) /(1-z))=\left(\sum_{i=0}^{\infty} c_{2 i+1} z^{2 i+1}\right)\left(\sum_{i=0}^{k} a_{j} z^{j}\right)=\sum_{i=0}^{\infty} b_{i} z^{i}
$$

(observe that $1_{n}((1+z) /(1-z))$ is an odd function that is holomorphic at $\left.z=0\right)$, where

$$
\begin{aligned}
& b_{0}=0 \\
& b_{1}=c_{1} a_{0} \\
& b_{2}=c_{1} a_{1} \\
& \cdots \\
& b_{2 i}=a_{1} c_{2 i-1}+a_{3} c_{2 i-3}+\cdots+a_{2 i-1} c_{1} \\
& b_{2 i+1}=a_{2} c_{2 i-1}+a_{4} c_{2 i-3}+\cdots+a_{2 i} c_{1}
\end{aligned}
$$

for $i=1,2,3, \ldots$, and $a_{j}:=0$ for $j>k$.

That $b_{k} \neq 0$ follows now from the fact that $a_{j}$ are all nonzero and of the same sign (see above) and that the $c_{2 i+1}$ are strictly positive.

To see the latter we compute the $c_{2 i+1}$ explicitly. Define

where

$$
\eta(z):=\ln ((1+z) /(1-z))=\sum_{j=0}^{\infty} c_{2 j+1} z^{j+1},
$$

$$
c_{2 j+1}=\eta^{(2 j+1)}(0) /(2 j+1) ! .
$$

We have $\eta^{\prime}(z)=2 /\left(1-z^{2}\right)$. Define

$$
H(z):=2 /\left(1-z^{2}\right)=\sum_{j=0}^{\infty} d_{2 j^{2}}{ }^{2 j},
$$

where $d_{2 j}=H^{(2 j)}(0) /(2 j)$ !. (Note that $H(z)$ is an even function and that it is holomorphic at $z=0$.)

From

$$
\begin{aligned}
\left(1-z^{2}\right) H(z) & =\left(1-z^{2}\right) \sum_{j=0}^{\infty} d_{2 j^{2 j}} \\
& =d_{0}+\sum_{j=0}^{\infty}\left(d_{2 j+2}-d_{2 j}\right) z^{2 j+2}=2
\end{aligned}
$$

it follows by induction that

$$
d_{2 j}=2 \quad(j=0,1,2, \ldots) .
$$

Hence, we obtain

$$
2=d_{2 j}=H^{(2 j)}(0) /(2 j) !=\eta^{(2 j+1)}(0) /(2 j) !,
$$


i.e.

$$
\eta^{(2 j+1)}(0)=2 *(2 j) !
$$

From (3.7) it follows that

$$
c_{2 j+1}=2 /(2 j+1)
$$

which completes the proof.

Remark. Henrici in his theorem on the maximum order of zero-stable ELMMs distinguishes between the case where the step-number is even and odd. This is because zero-stability allows for zeros of $\zeta(r)$ of modulus 1 , whereas infinite-stability does not (for zeros of $\hat{\sigma}(r)$ ).

The intersections of the regions of absolute instability of strongly infinite-stable ILMMs and the negative real line are given in the specifications of ILMMs in the following chapter. Plots of the regions of absolute instability can be found in [2] . There also an approach to compromising between strong instability and the size of the region of absolute instability is described.

4. Specification of Inverse Linear Multistep Methods. The following is a list of inverse linear $k$-step methods, of order $k-2$, for $k=3,4,5,6$, with free parameters that give complete control over the coefficients and, thus, over the zeros of $\hat{\sigma}(r)$. Also given is the error constant in terms of these parameters, and the maximum value of $-h \lambda$ (real), $h \lambda_{\mathrm{m} \text { in }}$, say, for which the zeros of the stability polynomial have modulus $\geqslant 1$, if the method is the unique strongly infinite-stable method.

$k=3$

$$
\begin{aligned}
f_{n+3}= & -\hat{\beta}_{2} f_{n+2}-\hat{\beta}_{1} f_{n+1}-\hat{\beta}_{0} f_{n} \\
& +\left(\left(5+3 \hat{\beta}_{2}+\hat{\beta}_{1}-\hat{\beta}_{0}\right) y_{n+2}+\right. \\
& +\left(-8-4 \hat{\beta}_{2}+4 \hat{\beta}_{0}\right) y_{n+1} \\
& \left.+\left(3+\hat{\beta}_{2}-\hat{\beta}_{1}-3 \hat{\beta}_{0}\right) y_{n}\right) /(2 h)
\end{aligned}
$$

order of the method: 1

error constant: $\hat{C}_{3}=\left(-11-2 \hat{\beta}_{2}+\hat{\beta}_{1}-2 \hat{\beta}_{0}\right) / 6$

$h \lambda_{\text {min }}=8$

$\underline{k=4}$

$$
\begin{aligned}
f_{n+4}=- & \hat{\beta}_{3} f_{n+3}-\hat{\beta}_{2} f_{n+2}-\hat{\beta}_{1} f_{n+1}-\hat{\beta}_{0} f_{n} \\
& +\left(\left(26+11 \hat{\beta}_{3}+2 \hat{\beta}_{2}-\hat{\beta}_{1}+2 \hat{\beta}_{0}\right) y_{n+3}\right. \\
& +\left(-57-18 \hat{\beta}_{3}+3 \hat{\beta}_{2}+6 \hat{\beta}_{1}-9 \hat{\beta}_{0}\right) y_{n+2} \\
& +\left(42+9 \hat{\beta}_{3}-6 \hat{\beta}_{2}-3 \hat{\beta}_{1}+18 \hat{\beta}_{0}\right) y_{n+1} \\
& \left.+\left(-11-\hat{\beta_{3}}+\hat{\beta}_{2}-2 \hat{\beta}_{1}-11 \hat{\beta}_{0}\right) y_{n}\right) /(6 h)
\end{aligned}
$$

order of the method: 2

error constant: $\hat{C}_{4}=\left(-25-3 \hat{\beta}_{3}+\hat{\beta}_{2}-\hat{\beta}_{1}+3 \hat{\beta}_{0}\right) / 12$

$h \lambda_{\min }=68 / 3$ 
$k=5$

$$
\begin{aligned}
f_{n+5}=-\hat{\beta}_{4} f_{n+4}-\hat{\beta}_{3} f_{n+3}-\hat{\beta}_{2} f_{n+2}-\hat{\beta}_{1} f_{n+1}-\hat{\beta}_{0} f_{n} \\
+\left(\left(77+25 \hat{\beta}_{4}+3 \hat{\beta}_{3}-\hat{\beta}_{2}+\hat{\beta}_{1}-3 \hat{\beta}_{0}\right) y_{n+4}\right. \\
+\left(-214-48 \hat{\beta}_{4}+10 \hat{\beta}_{3}+8 \hat{\beta}_{2}-6 \hat{\beta}_{1}+16 \hat{\beta}_{0}\right) y_{n+3} \\
+\left(234+36 \hat{\beta}_{4}-18 \hat{\beta}_{3}+18 \hat{\beta}_{1}-36 \hat{\beta}_{0}\right) y_{n+2} \\
+\left(-122-16 \hat{\beta}_{4}+6 \hat{\beta}_{3}-8 \hat{\beta}_{2}-10 \hat{\beta}_{1}+48 \hat{\beta}_{0}\right) y_{n+1} \\
\left.\quad+\left(25+3 \hat{\beta}_{4}-\hat{\beta}_{3}+\hat{\beta}_{2}-3 \hat{\beta}_{1}-25 \hat{\beta}_{0}\right) y_{n}\right) /(
\end{aligned}
$$

order of the method: 3

error constant: $\hat{C}_{5}=\left(-137-12 \hat{\beta}_{4}+3 \hat{\beta}_{3}-2 \hat{\beta}_{2}+3 \hat{\beta}_{1}-\hat{\beta} 12_{0}\right) / 60$

$h \lambda_{\min }=56$

$k=6$

$$
\begin{aligned}
f_{n+6}=\hat{\beta}_{5} f_{n+5}-\hat{\beta}_{4} f_{n+4}-\hat{\beta}_{3} f_{n+3}-\hat{\beta}_{2} f_{n+2}-\hat{\beta}_{1} f_{n+1}-\hat{\beta}_{0} f_{n} \\
+\left(\left(522+137 \hat{\beta}_{5}+12 \hat{\beta}_{4}-3 \hat{\beta}_{3}+2 \hat{\beta}_{2}-3 \hat{\beta}_{1}+12 \hat{\beta}_{0}\right) y_{n+5}\right. \\
\quad+\left(-1755-300 \hat{\beta}_{5}+65 \hat{\beta}_{4}+30 \hat{\beta}_{3}-15 \hat{\beta}_{2}+20 \hat{\beta}_{1}-75 \hat{\beta}_{0}\right) y_{n+4} \\
+\left(2540+300 \hat{\beta}_{5}-120 \hat{\beta}_{4}+20 \hat{\beta}_{3}+60 \hat{\beta}_{2}-60 \hat{\beta}_{1}+200 \hat{\beta}_{0}\right) y_{n+3} \\
+\left(-1980-200 \hat{\beta}_{5}+60 \hat{\beta}_{4}-60 \hat{\beta}_{3}-20 \hat{\beta}_{2}+120 \hat{\beta}_{1}-300 \hat{\beta}_{0}\right) y_{n+2} \\
+\left(810+75 \hat{\beta}_{5}-20 \hat{\beta}_{4}+15 \hat{\beta}_{3}-30 \hat{\beta}_{2}-65 \beta_{1}+300 \hat{\beta}_{0}\right) y_{n+1} \\
\left.\quad+\left(-137-12 \hat{\beta}_{5}+3 \hat{\beta}_{4}-2 \hat{\beta}_{3}+3 \hat{\beta}_{2}-12 \hat{\beta}_{1}-137 \hat{\beta}_{0}\right) y_{n}\right) /(60 h)
\end{aligned}
$$

order of the method: 4

error constant: $\hat{C}_{6}=\left(-147-10 \hat{\beta}_{5}+2 \hat{\beta}_{4}-\hat{\beta}_{3}+\hat{\beta}_{2}-2 \hat{\beta}_{1}+10 \hat{\beta}_{0}\right) / 60$ $h \lambda_{\min }=1936 / 15$

5. Comparison of Explicit Linear Multistep Methods and Inverse Linear Multistep Methods. In the following table some properties of LMMs and ILMMs are compared:

ELMM

ILMM

minimum step-number of consistent method

minimum step-number of zero-

(resp. infinite-) stable consistent

method

maximum order of zero- (resp.

infinite-) stable $k$-step method

$k$

$k-2$ 
6. Application of Inverse Linear Multistep Methods of Certain Stiff Systems of Ordinary Differential Equations. In [1] the application of so-called CDS (Correction in the Dominant Space) schemes to certain stiff systems is described. These separably stiff systems are characterized by the occurrence of a few (s, say) large negative eigenvalues of the Jacobian which dominate the rest of the eigenvalues clustered about the origin.

A special CDS scheme, based on ILMMs, is given by

$$
\hat{y}_{n+k}=\sum_{j=0}^{k-1}\left(-\alpha_{j} y_{n+j}+h \beta_{j} f_{n+j}\right)
$$

$$
\begin{gathered}
\hat{f}_{n+k}=\sum_{j=0}^{k-1}\left(-h^{-1} \hat{\alpha}_{j} y_{n+j}+\hat{\beta}_{j} f_{n+j}\right), \\
y_{n+k}=\hat{y}_{n+k}+\sum_{j=1}^{s} \xi_{n+k}^{(j)} c_{n+k}^{(j)},
\end{gathered}
$$

where the $c_{n+k}^{(j)}(j=1,2, \ldots, s)$ are the eigenvectors corresponding to the dominant eigenvalues of the Jacobian, evaluated at $\left(x_{n+k}, y_{n+k}\right)$. The corresponding eigenvalues and left eigenvectors are denoted by $\lambda_{n+k}^{(j)}$ and $d_{n+k}^{(j)}$, respectively.

The scalar correction factors $\xi_{n+k}^{(j)}(j=1,2, \ldots, s)$ are chosen such that

$$
\left\langle d_{n+k}^{(j)}, f\left(x_{n+k}, y_{n+k}\right)\right\rangle=\left\langle d_{n+k}^{(j)}, \hat{f}_{n+k}\right\rangle, \quad j=1,2, \ldots, s,
$$

where $\langle a, b\rangle=a^{T} b$, the standard inner product of vectors. (We normalize the eigenvectors such that $\left\langle d_{n+k}^{(i)}, c_{n+k}^{(j)}\right\rangle=\delta_{i j}, i, j=1,2, \ldots, s, \delta_{i j}$ the Kronecker delta, see [1].)

If the initial value problem (1.2) is defined by the linear function

$$
f(x, y)=A(x) y+g(x)
$$

(where $A(x)$ is an $m \times m$ matrix function) then the correction factors can be computed explicitly, giving:

$$
\xi_{n+k}^{(j)}=-\left\langle d_{n+k}^{(j)}, y_{n+k}\right\rangle-\frac{\left\langle d_{n+k}^{(j)}, g\left(x_{n+k}\right)-\hat{f}_{n+k}\right\rangle}{\lambda_{n+k}^{(j)}}, \quad j=1,2, \ldots, s
$$

For the general, nonlinear, case it is suggested in [2] to compute the $\xi_{n+k}^{(j)}$ by the iterative procedure

$$
\left[0 \xi_{n+k}^{(t)}=\xi_{n+k-1}^{(t)}\right.
$$

$$
[r+1] \xi_{n+k}^{(t)}
$$

$$
\begin{array}{r}
{[r] \xi_{n+k}^{(t)}-\frac{1}{\lambda_{n+k}^{(t)}}\left\langle d_{n+k}^{(t)}, f\left(x_{n+k}, \hat{y}_{n+k}+\sum_{i=1}^{s}|r|_{\xi_{n+k}}^{(i)} c_{n+k}^{(i)}\right)-\hat{f}_{n+k}\right\rangle} \\
t=1,2, \ldots, s ; r=0,1,2, \ldots
\end{array}
$$


In the linear case the above iteration scheme terminates after one step, and reduces to (6.4).

Some properties of the CDS scheme (6.1) are covered by the two following theorems. For the first one compare [1].

For both theorems we note that the dominant components of a vector $y$ are given by $\left\langle d_{n+k}^{(i)}, y\right\rangle, i=1,2, \ldots, s$ (see [1]).

THEOREM 2. The CDS scheme (6.1), with a rational basic method, and the correction factors given by (6.2) is dominantly stable, provided $h \lambda^{(i)} \in R_{p}, i=1,2$, $\ldots, s$, where $R_{p}$ is the region of absolute stability of (1.5).

Proof. If (6.1) is applied to $y^{\prime}=A y, A$ a constant separably stiff matrix, with correction factors defined by (6.2), then we obtain for $i=1,2, \ldots, s$ :

$$
\left\langle d^{(i)}, y_{n+k}\right\rangle=\left(\lambda^{(i)}\right)^{-1}\left(h^{-1} \sum_{j=0}^{k-1} \hat{\alpha}_{j}\left\langle d^{(i)}, y_{n+j}\right\rangle-\sum_{j=0}^{k-1} \hat{\beta}_{j}\left\langle d^{(i)}, \hat{f}_{n+j}\right\rangle\right),
$$

i.e. the $i$ th component of $y_{n+k}$ is obtained by applying (1.5) to the scalar IVP with starting values

$$
\varphi_{\mu}^{(i)}=\left\langle d^{(i)}, y_{\mu}\right\rangle, \quad \mu=0,1, \ldots, k-1 .
$$

Thus, $\left\langle d^{(i)}, y_{n}\right\rangle=\varphi_{n}^{(i)} \rightarrow 0$ as $n \rightarrow \infty$, for $i=1,2, \ldots, s$, if $h \lambda^{(i)} \in R_{p}$. This together with Theorem 1 in [1] proves the result.

Since, in the context of separably stiff systems, we are interested in large $\left|h \lambda^{(i)}\right|, i=1,2, \ldots, s$, it is appropriate to employ an infinite-stable ILMM for the computation of $f_{n+k}$ in (6.1(ii)). The region of absolute instability of infinitestable ILMMS may be fairly large, but, in practical applications, $h \lambda^{(i)}$ will usually be outside the region of absolute instability, because we assume that the dominant eigenvalues are large in modulus compared with the subdominant ones.

THEOREM 3. If the initial value problem (1.2) is defined by the linear function (6.3), then the dominant components of the local error of the CDS scheme (6.1) with correction factors given by (6.2) are of $O\left(h^{q+1}\right)$, where $q$ is the order of (1.5).

Proof. We can write

$$
g(x)=-A(x) y(x)+y^{\prime} c(x)
$$

(where $y(x)$ is the exact solution of (1.2)) and obtain for $i=1,2, \ldots, s$

$$
\begin{aligned}
\left\langle d_{n+k}^{(i)}, \hat{f}_{n+k}\right\rangle & =\left\langle d_{n+k}^{(i)}, f_{n+k}\right\rangle \quad(\text { by }(6.2)) \\
& =\left\langle d_{n+k}^{(i)}, A\left(x_{n+k}\right) y_{n+k}-A\left(x_{n+k}\right) y\left(x_{n+k}\right)+y^{\prime}\left(x_{n+k}\right)\right\rangle \\
& =\lambda_{n+k}^{(i)}\left\langle d_{n+k}^{(i)}, y_{n+k}\right\rangle-\lambda_{n+k}^{(i)}\left\langle d_{n+k}^{(i)}, y\left(x_{n+k}\right)\right\rangle+\left\langle d_{n+k}^{(i)}, y^{\prime}\left(x_{n+k}\right)\right\rangle
\end{aligned}
$$

i.e.

$$
\begin{aligned}
& \left\langle d_{n+k}^{(i)}, y_{n+k}\right\rangle \\
& \quad=\frac{1}{\lambda_{n+k}^{(i)}}\left(\left\langle d_{n+k}^{(i)}, \hat{f}_{n+k}\right\rangle+\left\langle d_{n+k}^{(i)}, y\left(x_{n+k}\right)\right\rangle-\left\langle d_{n+k}^{(i)}, y^{\prime}\left(x_{n+k}\right)\right\rangle\right) ;
\end{aligned}
$$

and hence, 


$$
\begin{gathered}
\left\langle d_{n+k}^{(i)}, y\left(x_{n+k}\right)-y_{n+k}\right\rangle=-\frac{1}{\lambda_{n+k}^{(i)}}\left(\left\langle d_{n+k}^{(i)}, \hat{f}_{n+k}\right\rangle-\left\langle d_{n+k}^{(i)}, y^{\prime}\left(x_{n+k}\right)\right\rangle\right) \\
=-\frac{1}{\lambda_{n+k}^{(i)}}\left(\left\langle d_{n+k}^{(i)}, \hat{f}_{n+k}-f\left(x_{n+k}, y\left(x_{n+k}\right)\right)\right\rangle\right) \\
=O\left(h^{q+1}\right) \text { by the definition of } q
\end{gathered}
$$

which completes the proof.

Remarks. For the linear problem it is trivial that the subdominant components of the local truncation error are of $O\left(h^{p+1}\right)$ where $p$ is the order of the ELMM employed in (6.1(i)).

For the nonlinear problem the dominant and subdominant components of the local truncation error are interdependent.

TABLE 1. Simple scalar numerical example

\begin{tabular}{rrrr}
$n$ & $x_{n}$ & \multicolumn{1}{c}{$y_{n}$} & \multicolumn{1}{c}{$y_{n}-y\left(x_{n}\right)$} \\
3 & 0.3 & 0.955 & $2.98 \mathrm{E}-7$ \\
4 & 0.4 & 0.921 & $4.76 \mathrm{E}-7$ \\
5 & 0.5 & 0.878 & $6.51 \mathrm{E}-7$ \\
10 & 1.0 & 0.540 & $1.39 \mathrm{E}-6$ \\
15 & 1.5 & 0.077 & $1.79 \mathrm{E}-6$ \\
20 & 2.0 & -0.416 & $1.75 \mathrm{E}-6$ \\
25 & 2.5 & -0.801 & $1.28 \mathrm{E}-6$ \\
30 & 3.0 & -0.990 & $5.02 \mathrm{E}-7$ \\
35 & 3.5 & -0.936 & $-4.03 \mathrm{E}-7$ \\
40 & 4.0 & -0.654 & $-1.21 \mathrm{E}-6$ \\
45 & 4.5 & -0.211 & $-1.72 \mathrm{E}-6$ \\
50 & 5.0 & 0.284 & $-1.81 \mathrm{E}-6$ \\
100 & 10.0 & -0.839 & $-7.78 \mathrm{E}-7$ \\
200 & 20.0 & 0.408 & $-1.55 \mathrm{E}-6$ \\
300 & 30.0 & 0.154 & $1.83 \mathrm{E}-6$ \\
1000 & 100.0 & 0.862 & $-1.13 \mathrm{E}-6$
\end{tabular}

\section{Numerical Examples.}

Example 1. Consider the scalar problem

$$
y(x)=\left(y^{\prime}+\sin (x)\right) / \lambda+\cos (x), \quad y^{\prime}(0)=0,
$$

with exact solution $y(x)=\cos (x)$. This example has been chosen because of its simplicity. It can be transformed into the form (1.2).

If $\lambda$ is large and negative, however, an implicit linear multistep method would have to be employed, if the numerical solution is to be stable and the step-length $h$ is not to be excessively small. Contrastingly, the application of an ILMM to (7.1) is fully explicit.

Table 1 contains numerical results, with $\lambda=-10^{4}$, and using the strongly in- 
finite-stable ILMM

$$
f_{n+3}=h^{-1}\left(5 y_{n+2}-8 y_{n+1}+3 y_{n}\right)
$$

with step-length $h=0.1$, and exact starting values.

We observe that, although there is an initial rise of the global error, the results are stable, and the accuracy is about $10^{-6}$. The errors do not alternate in sign as would be typical for instability. Because of the factor $1 / \lambda$ in (7.1) the accuracy in the $f_{n}$ is only about $10^{-2}$. However, usually one will be more interested in the approximations of $y(x)$ than in those of its derivative.

Example 2. Enright et al. in [6] give the following separably stiff initial value problem, arising in insulator physics:

$$
\begin{aligned}
& { }^{1} y^{\prime}=-{ }^{1} y+10^{8} *^{3} y\left(1-{ }^{1} y\right), \quad{ }^{1} y(0)=1, \\
& \text { (7.2(ii)) } \quad{ }^{2} y^{\prime}=10 *^{2} y+3 * 10^{7} *^{3} y\left(1-{ }^{2} y\right), \quad{ }^{2} y(0)=0, \\
& \text { (7.2(iii)) }{ }^{3} y^{\prime}=-{ }^{1} y^{\prime}-{ }^{2} y^{\prime} \text {, } \\
& { }^{3} y(0)=0, \quad x \in[0,1] \text {. }
\end{aligned}
$$

The Jacobian $A(x, y)$ of this system is singular for all $(x, y)$ (this is implied by (7.2(iii)); thus one of the eigenvalues is zero. On the exact solution curve the second eigenvalue decreases from -1 to -8.6 , the third and dominant one decreases from $-3 * 10^{7}$ to approximately $-4 * 10^{7}$, as $x$ increases from 0 to 1 .

The problem (7.2) was tackled using the CDS scheme (6.1), with correction factors defined by (6.2) and computed by (6.5), where

$$
\hat{f}_{n+k}=\frac{1}{6 h}\left(26 y_{n+3}-57 y_{n+2}+42 y_{n+1}-11 y_{n}\right) \text {. }
$$

Thus, $\hat{f}_{n+k}$ is defined by a strongly infinite-stable ILMM. The step-length was taken to be $h=0.01$.

For comparison purposes an "exact" solution was computed using the standard 4th order Runge-Kutta method with the very small step-length $h=5 * 10^{-8}$, which was required by stability.

The starting values were chosen to be the "exact" values $y\left(x_{1}\right), y\left(x_{2}\right), y\left(x_{3}\right)$, $y\left(x_{4}\right)$. The initial value $y\left(x_{0}\right)$ was not used because it lies in the transient phase (see [2]; there also alternative ways of finding starting values may be found).

The linear multistep method employed in (6.1(i)) is the standard fourth order Adams-Bashforth method.

The following measures of the maximum correction factor, and the maximum dominant and subdominant error, respectively, are given by

$$
\begin{aligned}
M C & =\max _{5 \leqslant n \leqslant 100}\left|\xi_{n}^{(1)}\right|, \\
M D & =\max _{5 \leqslant n \leqslant 100}\left|\left\langle d_{n}^{(1)}, y\left(x_{n}\right)-y_{n}\right\rangle\right| \\
M S & =\max _{5 \leqslant n \leqslant 100}\left\|y\left(x_{n}\right)-y_{n}-\left\langle d_{n}^{(1)}, y\left(x_{n}\right)-y_{n}\right\rangle c_{n}^{(1)}\right\|_{\infty} .
\end{aligned}
$$


Thus, $M D$ gives the maximum error in the dominant component of the numerical solution, and $M S$ gives the infinity norm of the error after the dominant component has been subtracted. The results are

$$
M C=1.18 E-11, \quad M D=1.58 E-17, \quad M S=1.60 E-6 .
$$

The numerical solution is stable, and the results are accurate. The dominant accuracy is even close to the working accuracy of the machine (the DEC 10 of the University of Dundee), the computations were carried out in double precision carrying 19 digits.

Note that this example is extremely difficult numerically. A treatment with conventional methods would require the use of fully implicit methods, whereas here the implicitness is reduced to the scalar problem of finding the single correction factor $\xi^{(1)}$.

Conclusions. A class of method has been proposed, that can be used with advantage for certain unconventional types of initial value problems, and that is particularly efficient if applied in conjunction with the CDS technique to the extremely difficult separably stiff initial value problems.

Acknowledgements. The material described in this paper is part of the results of a Ph.D. project carried out at the University of Dundee, Scotland. I am indebted to my supervisor, J. D. Lambert, for many stimulating discussions, and to the German National Scholarship Foundation for their generous financial support. The research was also supported financially by the University of Dundee.

Department of Mathematics

University of Utah

Salt Lake City, Utah 84112

1. P. ALFELD \& J. D. LAMBERT, "Correction in the dominant space: A numerical technique for a certain class of stiff initial value problems," (Dundee Report NA 18, August 1976, Univ. of Dundee), Math. Comp., v. 31, 1977, pp. 922-938.

2. P. ALFELD, Correction in the Dominant Space: A New Technique for the Numerical Solution of Certain Stiff Initial Value Problems, Ph.D. Thesis, Univ. of Dundee, Dundee DD1 4HN, Scotland, 1977.

3. B. L. EHLE, On Padé Approximations to the Exponential Function and A-Stable Methods for the Numerical Solution of Initial Value Problems, Univ. of Waterloo, Department of Applied Analysis and Computer Science, Research Report No. CSRR 2010, 1969.

4. W. E. ENRIGHT, T. E. HULL \& B. LINDBERG, "Comparing numerical methods for stiff systems of o.d.e.s," $B I T$, v. 15, 1975, pp. 10-48.

5. P. HENRICI, Discrete Variable Methods in Ordinary Differential Equations, Wiley, New York and London, 1972.

6. J. D. LAMBERT, Computational Methods in Ordinary Differential Equations, Wiley, New York and London, 1973. 